\title{
A universal drift-diffusion simulator and its application to OLED simulations
}

\author{
Francesco Santoni, Thomas Brown, Francesca Brunetti, Sara Pescetelli, \\ Andrea Reale, Aldo Di Carlo and Matthias Auf der Maur \\ Department of Electronic Engineering \\ University of Rome "Tor Vergata" \\ Rome 00133, Italy
}

Email: francesco.santoni@uniroma2.it, thomas.brown@uniroma2.it, francesca.brunetti@uniroma2.it, sara.pescetelli@uniroma2.it, reale@uniroma2.it, aldo.dicarlo@uniroma2.it, auf.der.maur@ing.uniroma2.it

\begin{abstract}
A universal simulation tool for electronic devices based on a semi-classical drift-diffusion (DD) model is presented. The core of the model is a fully-coupled system of Poisson equation for the electrostatic potential and drift-diffusion transport equations. Both charged and neutral (e.g. excitons) carriers are supported. One transport equation is associated to each carrier. The number of carriers can be set at user level. The equation system can be defined in 1, 2 and 3 dimensions, and it is solved using finite element methods (FEM). The simulator has many potential application, from simple semiconductors with electrons and holes transport, to far more complex device structures, such as the host-guest system of an OLED emitter layer including singlet and triplet excitons. The simulation of an OLED emitter layer is presented, including the thermally activated delayed fluorescence (TADF) effect.
\end{abstract}

\section{INTRODUCTION}

The simulation of electronic devices is nowadays a task that require to go far beyond the simple picture of semiconductors with electrons and holes transport in the conduction and valence bands respectively. Especially organic electronics is a continuously growing field ${ }^{[1]}$. Several applications have been intensively studied and developed, such as organic light emitting diodes (OLED), organic photovoltaics (OPV), organic field effect transistors (OFET). Amorphous and/or regular assembly of polymers/small molecules can form such materials which typically exhibit complex electronic properties. Besides electrons and holes transport, some device types include ions transport, such as dye-sensitized solar cell (DSSC) ${ }^{[3]}$ or resistive switching memories based on the formation of conductive filaments $^{[4]}$.

One of the most complex structure to simulate is the doped emitter layer (EML) of an OLED where the doping sites can also act as transport sites and not just as traps ${ }^{[5]}$. The simulator should account for electrons, holes, singlet and triplet excitons in the host material (the conductive matrix) and the guest material (the doping material), hence eight particles have to be included in the model. We present here the simulation of such a structure including also the TADF effect ${ }^{[6]}$.

\section{THE MODEL}

We developed the model within the multiscale simulation environment $\mathrm{TiberCAD}{ }^{[2]}$. The model has two types of equations. The Poisson equation for the electrostatic potential $\varphi$ :

$$
\nabla \cdot(\varepsilon \nabla \varphi)=-\rho
$$

where $\rho$ is the total charge (charged carriers plus fixed charges). The transport equation:

$$
-\nabla \cdot(\mu n \nabla \phi)=G-R
$$

where $\phi$ is the electrochemical potential of the carrier. The user can set, potentially, any number of carriers, each carrier with its own properties, and one transport equation is dinamically build for each carrier and added to the system. $\mu$ is the carrier mobility and $n$ its density. $G$ and $R$ are generation and recombination rates. Transport equations of charged carriers are coupled to the Poisson equation through the density $n$ entering in the total charge $\rho$. Transport equations are coupled among them through $G$ and $R$ associated to different processes, e.g. charge carriers transferred between host and guest, electrons and holes recombining into excitons, inter-system crossing (ISC) between singlets and triplets etc.

The electrochemical potential has been chosen as the dependent variable for several reason. Using e.c. potential, the transport equation has the general form (2) which is valid for both charged and neutral carriers. Boundary conditions for Schottky contacts can be easily set. Since TiberCAD is a multiscale simulator, it is far more easier using e.c potentials to couple the DD model with atomistic/quantum mechanical calculations. The use of e.c. potentials provides a natural way to write recombination rates which are compatible with the Fermi-Dirac (FD) or the Bose-Einstein (BE) statistics of carriers. For example, accordingly with FD and BE statistics, the recombination rates of electrons (e) and holes (h) forming excitons ( $\mathrm{x}$ ) can be written as ( $f$ 's are the occupation functions):

$$
\begin{aligned}
R \propto & f_{e}^{F D}\left(1-f_{h}^{F D}\right)\left(1+f_{x}^{B E}\right)- \\
& -\exp \left[-\frac{\left(E_{e}-E_{h}\right)-E_{x}}{k_{B} T}\right]\left(1-f_{e}^{F D}\right) f_{h}^{F D} f_{x}^{B E}
\end{aligned}
$$




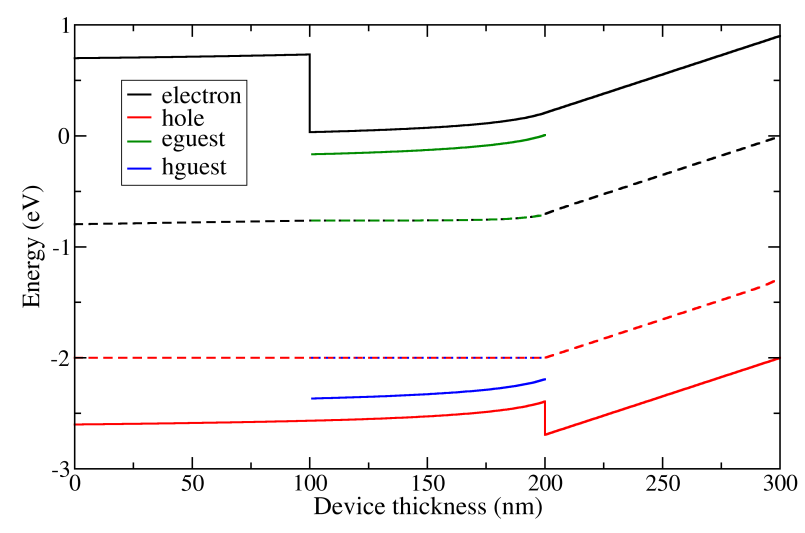

Fig. 1. Band structure of the simulated OLED with an applied bias of $2 \mathrm{~V}$. Dashed curves are the quasi-fermi levels

which, with some straightforward manipulation, is reduced to the simple form:

$$
\left\{1-\exp \left[\frac{\left(\phi_{e}-\phi_{h}\right)-\phi_{x}}{k_{B} T}\right]\right\} f_{e}^{F D}\left(1-f_{h}^{F D}\right)\left(1+f_{x}^{B E}\right)
$$

which is particularly feasible to be used in the simulation since it reduces naturally to zero at thermodynamic equilibrium.

\section{OLED SIMULATION}

We simulated a simple OLED structure. Device thickness is $300 \mathrm{~nm}$. The EML is in the middle and its thickness is $100 \mathrm{~nm}$. Guest material is included in the EML, with band edges inside the band gap of the host material. The band structure, with an applied bias of $2 \mathrm{~V}$, is shown in figure 1 . The quasi-fermi levels are also plotted (dashed curves). In figure 2, as an example, it is plotted (on the top) the recombination rate of electrons between host and guest. A positive sign means that electrons recombine (they "disappear" from the host material), while a negative sign means generation (electrons are generated on the guest material). Accordingly (bottom of figure 2) the electron current decreases in the host and increases in the guest.

In figure 3 it is shown the effect of the inter-system crossing allowing the exchange of singlet and triplet excitons. In materials where the triplet has a larger binding energy than the singlet, ISC effect makes the singlet decay into lower energy triplet states, while the opposite process is not possible. But if singlets and triplets have almost the same energy (we set exciton binding energy to be $0.3 \mathrm{eV}$ and $0.32 \mathrm{eV}$ respectively) the process can occur in both directions. But singlets have also a faster radiative decay rate than triplets. Hence what is expected in TADF OLEDs is that the singlet population will decrease because of radiative decay, and that the ISC will occur mainly from triplets to singlets. That is exactly what can be seen in figure 3 where the ISC rate is plotted for singlets and triplets in the guest material. Triplet ISC rate is positive all across the EML (they recombine) while singlet ISC rate is negative (they are generated - and then they decay radiatively,

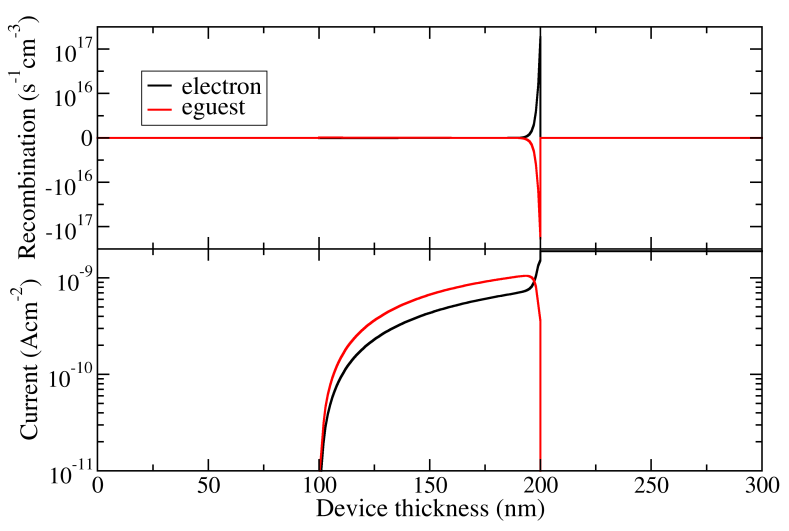

Fig. 2. Electron recombination rates between host and guest (top). Electrons current in host and guest (bottom)

hence the main light emission mechanism in TADF OLEDs is fluorescence and not phosphorescence).

\section{ACKNOWLEDGMENT}

We acknowledge the support of EU-H2020 project "MOSTOPHOS" (n. 646259)

\section{REFERENCES}

[1] F. Santoni, A. Gagliardi, M. Auf der Maur, A. Di Carlo, Org. Electron. 15 (2014) 1557

[2] M. Auf der Maur, IEEE Trans. on Electron. Devices, vol. 58, no. 5, 1425 (2011)

[3] A. Gagliardi, M. Auf der Maur, D. Gentilini, A. Di Carlo, J. Comput. Electron. 10 (2011) 424

[4] G. Niu et al., Sci. Rep. 6 (2016) 25757

[5] I. S. Park, S. R. Park, D. Y. Shin, J. S. Oh, W. J. Song, J. H. Yoon, Org. Electron. 11 (2010) 218

[6] Q. Zhang et al., Nature Photonics 8 (2014) 326

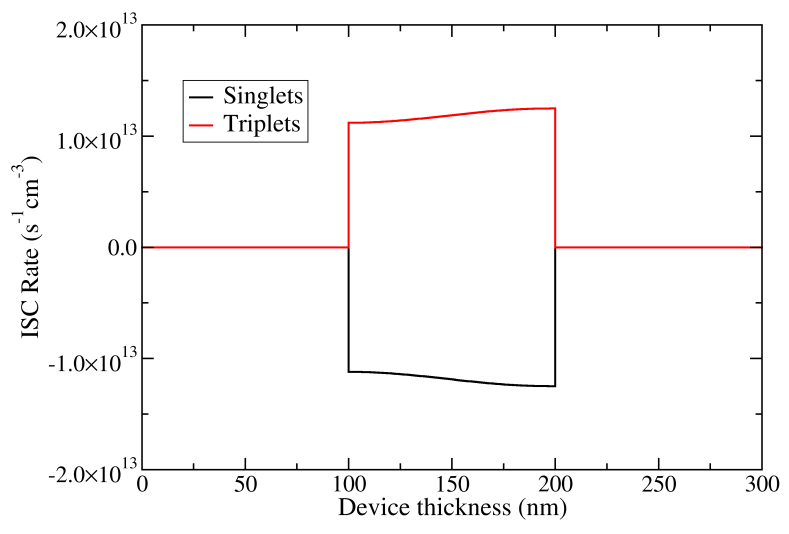

Fig. 3. Inter-system crossing rate between singlets and triplets in the guest material. Triplets recombine and singlets are generated by ISC 